\title{
Manejo Quirúrgico de Múltiples Condilomas de la Mucosa Oral en Paciente Bajo Terapia Antirretroviral de Gran Actividad
}

\author{
Surgical Management of Multiple Condylomas in the Oral Mucosa \\ in Patient Undergoing Highly Active Antiretroviral Therapy
}

Rodrigo Soto $\mathbf{R}^{1}$; Javier Pérez $\mathbf{A}^{1}$ \& Cristóbal Araya $\mathbf{S}^{2}$

SOTO, R. R.; PÉREZ, A. J. \& ARAYA, S. C. Manejo quirúrgico de múltiples condilomas de la mucosa oral en paciente bajo terapia antirretroviral de gran actividad. Int. J. Odontostomat., 12(1):93-98, 2018.

RESUMEN: El objetivo de este estudio fue reportar un caso clínico donde se realizó el manejo quirúrgico de múltiples condilomas de la mucosa oral en un paciente infectado por Virus de Inmunodeficiencia Humana (VIH) bajo Terapia Antirretroviral de Gran Actividad (TARGA). Hombre de 58 años en tratamiento por infección con VIH en TARGA hace 17 años, que acude al Servicio de Cirugía Maxilofacial del Hospital Barros Luco-Trudeau con múltiples lesiones verruciformes ubicadas en margen y cara dorsal de lengua, cara interna de ambas mejillas y labio inferior. Se realizó escisión quirúrgica de las lesiones de labio, cara dorsal de lengua y cara interna de mejilla del lado derecho, obteniéndose el diagnóstico histopatológico de condiloma. Tras 2 meses de realizar la cirugía se obtuvo recurrencia. La recurrencia de las lesiones puede originarse por la recrudescencia del virus latente adyacente al lecho quirúrgico y, por ello, deben considerarse otras alternativas de tratamiento. Por el impacto en la función, estética, potencial de contagio y malignización, es necesario su tratamiento.

PALABRAS CLAVE: condiloma acuminado; cirugía oral; Terapia Antirretroviral de Gran Actividad; VIH.

\section{INTRODUCCIÓN}

La Terapia Antirretroviral de Gran Actividad (TARGA) se basa en la combinación de dos o más drogas antirretrovirales para el tratamiento de la infección causada por el Virus de Inmunodeficiencia Humana tipo 1 (VIH-1) (Ray et al., 2010). Esta terapia incluye el uso de inhibidores de la transcriptasa reversa análogo de los nucléosidos, inhibidores no nucléosidos de la transcriptasa reversa y/o inhibidores de las aspártico proteasas combinado, a veces, con inhibidores de fusión (Barbaro et al., 2005).

La reconstitución inmunológica tras la utilización de TARGA ha permitido disminuir la mortalidad y morbilidad en pacientes con VIH (Arts \& Hazuda, 2012). Asimismo, limita la incidencia de infecciones oportunistas, incluyendo las lesiones orales relacionadas al VIH (Ortega et al., 2009). Estas comprenden: candiadisis oral, leucoplasia pilosa, Sarkoma de Kaposi, herpes simple tipo 1 y enfermedad periodontal (Ortega et al.; Taiwo \& Hassan, 2010).

Por otro lado, estudios han encontrado un aumento en la prevalencia de lesiones verruciformes de la mucosa oral en TARGA de larga data, principalmente asociadas al Virus Papiloma Humano (VPH) (Greenspan et al., 2001; King et al., 2002; Anaya-Saavedra et al., 2013). Entre estas, se pueden citar: papiloma escamoso oral, verruga vulgar, condiloma e hiperplasia epitelial focal (Anaya-Saavedra et al.).

Debido a que las lesiones orales producidas por VPH pueden afectar la estética y funcionalidad del paciente, ser contagiosas y exhibir potencial de malignización (Syrjänen et al., 2011), es que es importante su tratamiento. Se presenta el caso del manejo quirúrgico de un paciente $\mathrm{VIH}$ con diagnóstico de múltiples condilomas en la mucosa oral.

\footnotetext{
${ }^{1}$ Servicio de Cirugía Maxilofacial, Hospital Barros Luco-Trudeau, Santiago, Chile.

${ }^{2}$ Departamento de Patología Oral y Maxilofacial, Facultad de Odontología, Universidad Mayor, Santiago, Chile.
} 


\section{CASO CLÍNICO}

Hombre de 58 años acude al Servicio Maxilofacial del Hospital Barros Luco-Trudeau por desarrollo de múltiples lesiones orales de 8 meses de evolución. Presenta diagnóstico de infección por VIH y Virus Hepatitis B (VHB) hace 17 y 11 años, respectivamente. Antecedentes de parálisis facial periférica, leucoplasia pilosa, microhematuria, prurito, síndrome diarreico agudo y herpes zoster abdominal desarrollados el mismo año de la pesquisa de $\mathrm{VIH}$. Hábito tabáquico activo. Se han instaurado diversos protocolos de TARGA, presentándose múltiples fracasos y resistencia al tratamiento de delavirdine, efavirenz y neviparine, evidenciado por informe de resistencia a drogas antirretrovirales. Actualmente en terapia con esquema de tenofovir/ emtricitabina, darunavir/ritonavir y raltegravir, recuen- to de linfocitos TCD4 de 293 células/mL, carga viral indetectable y sin otras manifestaciones asociadas a la infección por VIH.

A la exploración se observan múltiples lesiones verruciformes en margen y cara dorsal de lengua, cara interna de ambas mejillas y labio inferior, extendiéndose incluso hacia la comisura y límite mucocutáneo (Fig. 1). Lesiones presentan diámetros diversos, desde $5 \mathrm{~mm}$ hasta $3 \mathrm{~cm}$, coloración de mucosa normal, textura pedregosa y tendencia a la coalescencia. Paciente relata 8 meses de evolución, incomodidad y problemas para alimentarse.

Con el diagnóstico presuntivo de condilomas de la mucosa oral, se realizó el manejo quirúrgico.

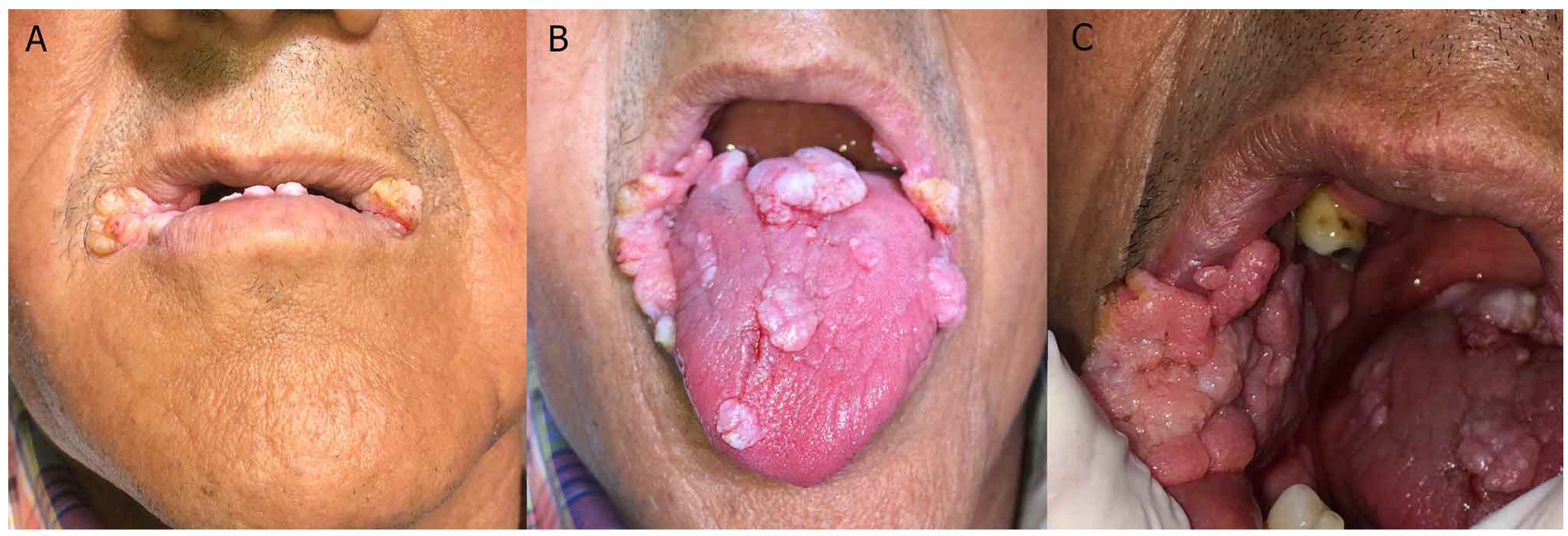

Fig. 1. A) Lesiones en labio inferior, B) cara dorsal de lengua, y C) cara interna de mejilla que se extienden comprometiendo comisura labial alcanzando el límite mucocutáneo.

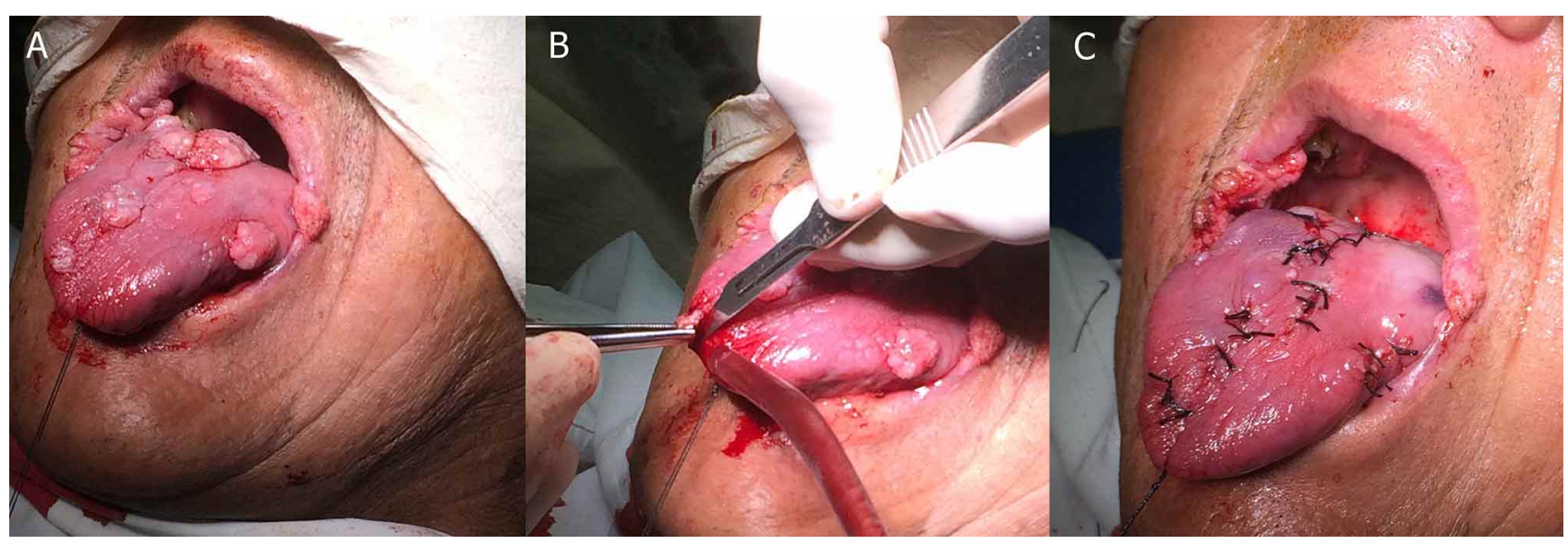

Fig. 2. A) Punto de rienda en vértice lingual, B) incisión con bisturí de lesiones en dorso de lengua, y C) lecho quirúrgico con suturas en posición. 
Bajo anestesia local, se llevó a cabo la extirpación de las lesiones ubicadas en el labio inferior, cara dorsal de la lengua y cara interna de mejilla derecha (Fig. 2). Se obtuvo un cierre primario de la mucosa lingual y labial con material de sutura seda 3-0, mientras que el lecho quirúrgico producto de la queilectomía labial, fue suturada por planos anatómicos tanto con monocryl como nylon 3-0 (Fig. 3). Posterior a la intervención se prescribieron analgésicos, antinflamatorios, antibióticos y colutorio de clorexhidina al 0,12 \%. Se envió una muestra obtenida de lengua y labio para su estudio histopatológico, confirmando el diagnóstico de condiloma de la mucosa oral (Fig. 4).

Se realizó un control a los 7 días de la intervención, donde se observó una evolución positiva de los lechos quirúrgicos, leve sintomatología dolorosa y sin signos de infección. Ya a los 2 meses tras la escisión de las lesiones se constató una reincidencia de las mismas ubicadas en labio, lengua y cara interna de mejilla, alcanzando en algunas regiones un tamaño y distribución similar al presentado antes del procedimiento (Fig. 5).

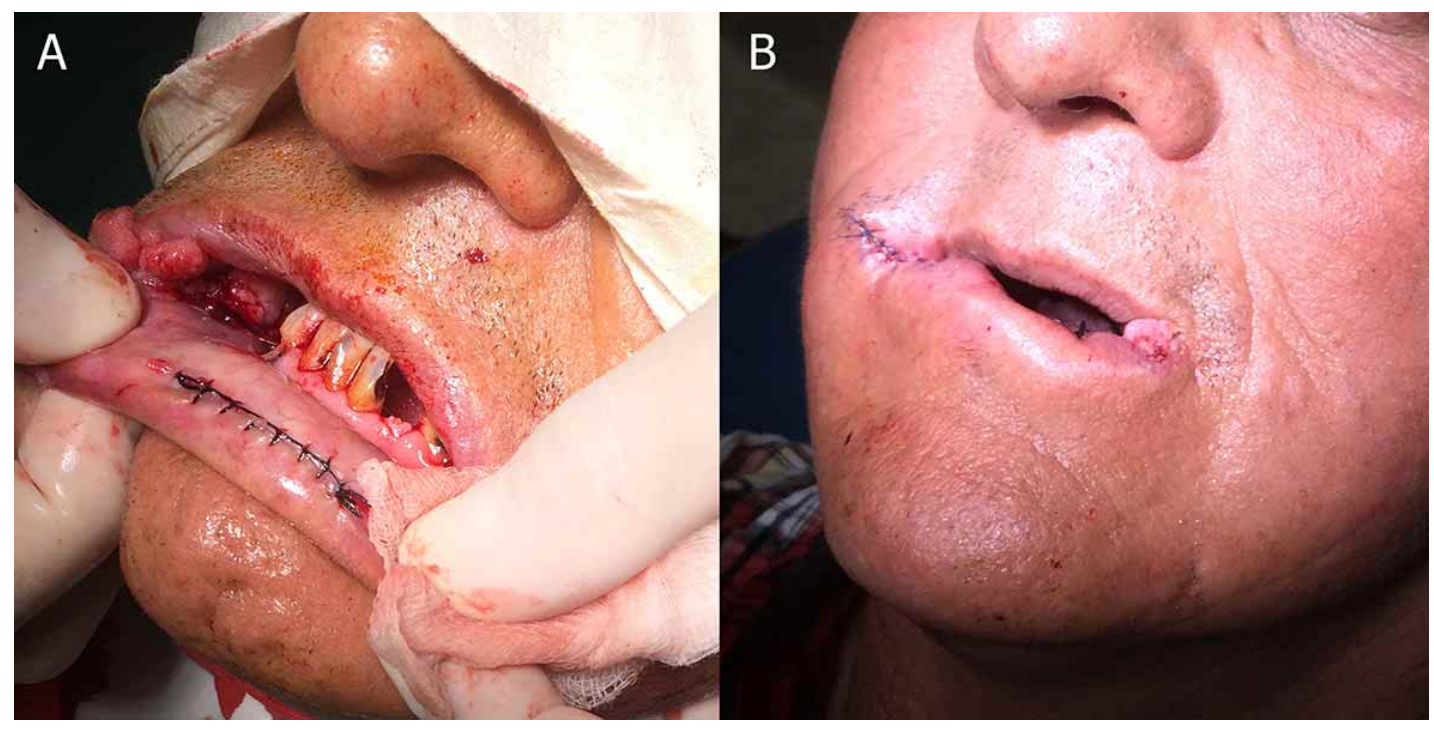

Fig. 3. A) Sutura continua en labio inferior y B) vista frontal del paciente en el postoperatorio inmediato. Nótese extensión de queilectomía.

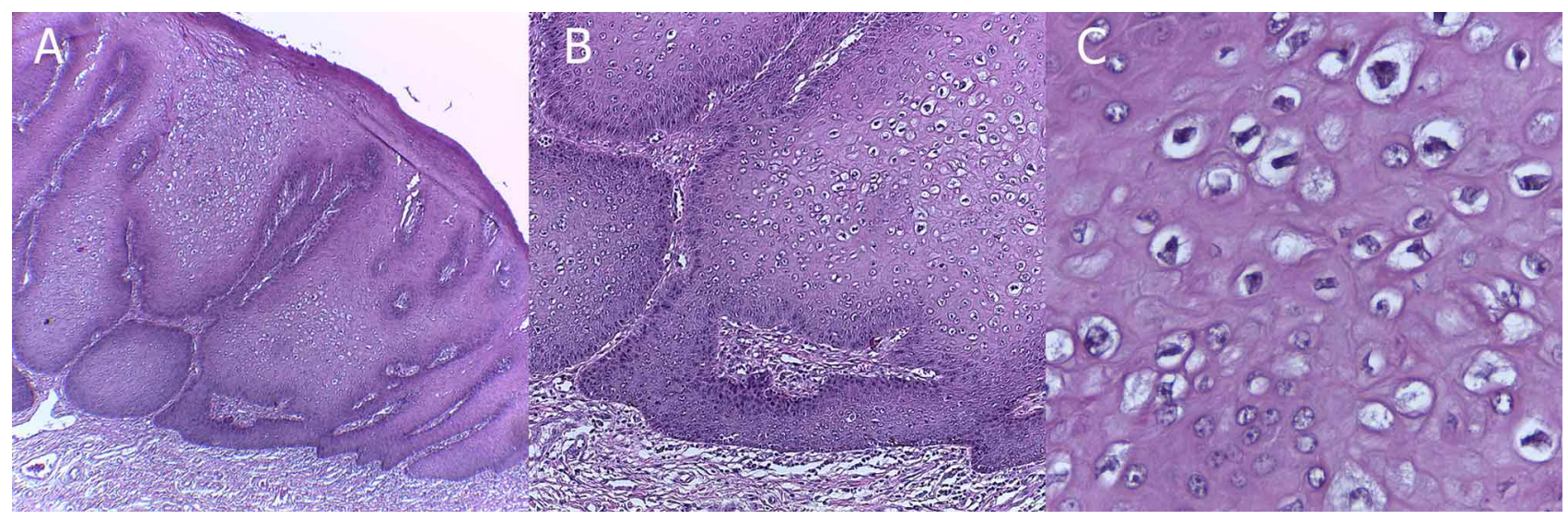

Fig. 4. A) Microfotografía corte histológico tinción hematoxilina-eosina 4x de muestra de mucosa oral con adyacente hiperplasia epitelial con hiperparaqueratosis y células de halo blanquecino, B) 10x. abundantes células de núcleo picnótico y halo blanquecino perinuclear, correspondientes a coilocitos en el estrato espinoso y C) 40x. estrato espinoso con células mitosoides y abundantes coilocitos. 


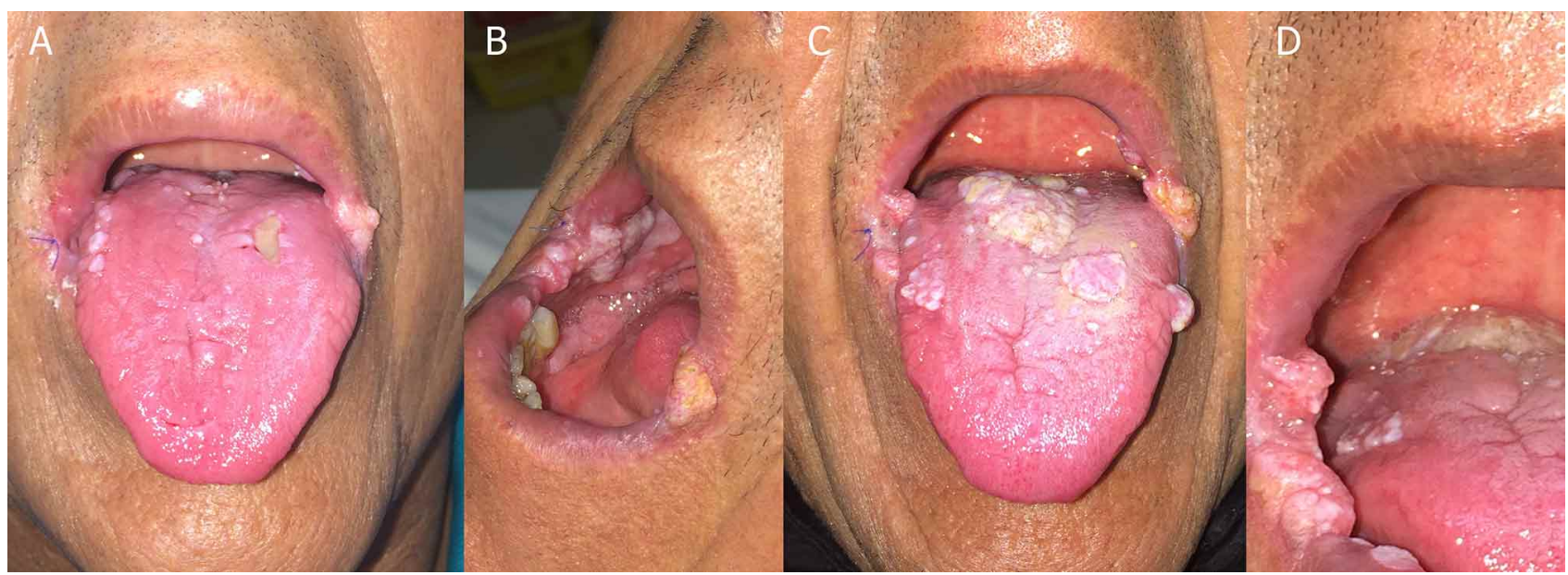

Fig. 5. A) Lesiones comenzando a reincidir a 1 mes de la cirugía en lengua y, B) comisura y cara interna de mejilla derecha,y C) a 2 meses en lengua y D) comisura labial derecha.

\section{DISCUSIÓN}

El Síndrome de Reconstitución Inmune (SRI) se caracteriza por un empeoramiento paradójico del estado clínico del paciente o la aparición de una nueva condición tras la iniciación de TARGA en aquellos infectados con VIH (Bosamiya, 2011). Si bien se han descrito sus manifestaciones, la patogénesis aún no se encuentra claramente identificada aunque se postula que estaría involucrada una activación exagerada del sistema inmune frente antígenos persistentes o patógenos viables (Müller et al., 2010).

Una nueva tendencia que se ha descrito recientemente corresponde a la aparición de lesiones verruciformes en la mucosa oral asociada al uso de TARGA (Greenspan et al.; Anaya-Saavedra et al.; King et al.). Existen estudios que han propuesto que sería una manifestación de SRI (Meys et al., 2010), mientras que otros sostienen que no existiría un efecto demostrable en la respuesta inmune local en este tipo de patologías (Lilly et al., 2005).

Una de las primeras investigaciones en registrar esta tendencia fue conducida por Greenspan et al. en donde se encontró que en pacientes en TARGA, si bien existía una disminución en patologías como candidiasis oral, leucoplasia pilosa y Sarcoma de Kaposi, se registró un aumento en enfermedades de las glándulas salivales y lesiones verruciformes, estas últimas alcanzando una frecuencia seis veces mayor.
En un estudio transversal realizado por AnayaSaavedra et al. se encontró una prevalencia de 6,9\% de lesiones verruciformes orales, identificándose que la edad (> 40 años), tiempo de tratamiento con TARGA (> 12 meses) y carga viral indetectable se constituyen como factores independientes asociados a su desarrollo. Estas variables de riesgo se condicen con las presentadas en nuestro caso clínico. Finalmente, en este estudio no se pesquizaron condilomas de la mucosa oral, lo cual podría sugerir una baja prevalencia dentro de las lesiones provocadas por VPH en pacientes en TARGA.

King et al. encontraron una prevalencia de 2,6 \% de lesiones verruciformes orales, asociadas a pacientes con una reducción en su carga viral. Además, identificaron como factor de riesgo la evidencia serológica de infección crónica o previa con VHB, lo que en nuestro caso también se encontraba diagnosticado.

Las lesiones verruciformes en el contexto de pacientes VIH en terapia con TARGA tienen las características de presentar una aparición súbita, con un curso rápido e incontrolado y ser refractarias a los tratamientos convencionales, siendo capaces de generar gran incomodidad y problemas estéticos. Hasta el momento, no se ha establecido una cura (Greenspan et al.).

Dentro de la gama de tratamientos clásicos para el manejo de estas patologías de la mucosa oral se encuentra la cirugía estandar, procedimiento encar- 
gado de la escisión de las lesiones. En nuestro caso se optó por esta modalidad de tratamiento combinando incisión con bisturí y electrobisturí. Sin embargo, se observó una recurrencia de la lesión en magnitud y distribución similar a la preoperatoria al cabo de 2 meses. Esto puede ser debido a que se generó una recrudesencia del virus latente adyacente al lecho quirúrgico, como lo ha establecido la literatura (Lipke, 2006).

Cabe considerar, entonces, otras alternativas de tratamiento, como la crioterapia, que se basa en el uso de criógenos como el nitrógeno líquido que alcanza una temperatura de $-196^{\circ} \mathrm{C}$ y su efecto terapéutico ocurre por la destrucción de queratinocitos infectados por VPH o a través de la inducción de una respuesta inflamatoria local, aunque el virus no es erradicado y también puede recurrir. Opciones como terapia laser $\left(\mathrm{CO}_{2}\right.$, Er:Yag, etc), terapia fotodinámica, uso de antimicóticos (bleomicina), agentes queratolíticos (ácido tricloroacético), inmunomoduladores (imiquimod), entre otros, constituyen también parte del arsenal terapéutico (Lipke; Curi et al., 2017).

El imiquimod se presenta como otra alternativa para el tratamiento del condiloma en la mucosa oral y puede actuar estimulando a las citoquinas, incluyendo interferon-a, interleukina-1 y 6 , factor de necrosis tumoral-a, factor estimulante de colonias de granulocitos y monocitos y factor estimulante de colonias de granulocitos. En un caso reportado por Curi et al., tras el fracaso terapéutico obtenido con criocirugía y aplicación de ácido tricloroacético, decidieron realizar la aplicación de imiquimod al $5 \%, 3$ veces a la semana en las noches durante 8 semanas, con lo que se obtuvo reducción progresiva y significativa de las lesiones, quedando sólo una pápula que fue removida con cirugía y se reportó que no ha existido recurrencia durante 2 años. Sin embargo, aquel caso difere del nuestro en que la presentación de la patología fue mucho más discreta, limitada solo al labio, por lo tanto, sería interesante observar el alcance terapéutico del imiquimod en una lesión de la cuantía como la presentada en este caso.

\section{CONCLUSIÓN}

La baja frecuencia de condilomas orales se enmarca dentro de una tendencia mayor de lesiones producidas por VPH en pacientes en TARGA, presentando un desafío para su tratamiento por la alta recurrencia. Sin embargo, el manejo se hace impera- tivo debido a la posibilidad de transformación maligna observada por algunas cepas de VPH en el territorio oral, además de ser contagiosas y producir, eventualmente, deformidad y consecuente impacto en la estética y funcionalidad del paciente.

SOTO, R. R.; PÉREZ, A. J. \& ARAYA, S. C. Surgical management of multiple condylomas in the oral mucosa in patient undergoing highly active antiretroviral therapy. Int. J. Odontostomat., 12(1):93-98, 2018.

ABSTRACT: The objective of this study was to report a clinical case in which surgical management of multiple condylomas in the oral mucosa was performed in a patient infected by Human Immunodeficiency Virus (HIV) who is under highly active antiretroviral therapy (HAART). A 58-yearold man, under HAART for 17 years for HIV infection, was admitted at the Maxillofacial Surgery Service at the Hospital Barros Luco-Trudeau as he was experiencing multiple verrucous lesions located on the lateral margin and dorsum of the tongue, as well as on the inner face of both cheeks and the lower lip. A surgical excision of the lesions on the lip, dorsum of the tongue and inner face of the right cheek was performed, where the histopathological diagnosis of condyloma was obtained. There was a recurrence two months after surgery. Recurrence of the lesions may be due to the recrudescence of the latent virus adjacent to the surgical bed and, therefore, other treatment alternatives should be considered. Treatment is necessary due to the impact on the function, aesthetics, and the potential to become contagious and malignant.

KEY WORDS: condylomata acuminate, oral surgery, antiretroviral therapy, highly active, HIV.

\section{REFERENCIAS BiBLIOGRÁFICAS}

Anaya-Saavedra, G.; Flores-Moreno, B.; García-Carrancá, A.; Irigoyen-Camacho, E.; Guido-Jiménez, M. \& Ramírez-Amador, V. HPV oral lesions in HIV-infected patients: the impact of longterm HAART. J. Oral Pathol. Med., 42(6):443-9, 2013.

Arts, E. J. \& Hazuda, D. J. HIV-1 antiretroviral drug therapy. Cold Spring Harb. Perspect. Med., 2(4):a007161, 2012.

Barbaro, G.; Scozzafava, A.; Mastrolorenzo, A. \& Supuran, C. T. Highly active antiretroviral therapy: current state of the art, new agents and their pharmacological interactions useful for improving therapeutic outcome. Curr. Pharm. Des., 11(14):1805-43, 2005.

Bosamiya, S. S. The immune reconstitution inflammatory syndrome. Indian J. Dermatol., 56(5):476-9, 2011.

Curi, D. S.; Leite-Ribeiro, P. M.; Torregrossa, V. R.; Vieira, V. C. \& Sarmento, V. A. Efficacy of imidazolquinoline on treatment of condyloma acuminatum of the buccal mucosa. Spec. Care Dent., 37(1):51-4, 2017.

Greenspan, D.; Canchola, A. J.; MacPhail, L. A.; Cheikh, B. \& Greenspan, J. S. Effect of highly active antiretroviral therapy on frequency of oral warts. Lancet, 357(9266):1411-2, 2001. 
SOTO, R. R.; PÉREZ, A. J. \& ARAYA, S. C. Manejo quirúrgico de múltiples condilomas de la mucosa oral en paciente bajo terapia antirretroviral de gran actividad. Int. J. Odontostomat., 12(1):93-98, 2018

King, M. D.; Reznik, D. A.; O'Daniels, C. M.; Larsen, N. M.; Osterholt, D. \& Blumberg, H. M. Human papillomavirus-associated oral warts among human immunodeficiency virus-seropositive patients in the era of highly active antiretroviral therapy: an emerging infection. Clin. Infect. Dis., 34(5):641-8, 2002.

Lilly, E. A.; Cameron, J. E.; Shetty, K. V.; Leigh, J. E.; Hager, S.; McNulty, K. M.; Cheeks, C.; Hagensee, M. E. \& Fidel, P. L. Jr. Lack of evidence for local immune activity in oral hairy leukoplakia and oral wart lesions. Oral Microbiol. Immunol., 20(3):154-62, 2005.

Lipke, M. M. An armamentarium of wart treatments. Clin. Med. Res., 4(4):273-93, 2006.

Meys, R.; Gotch, F. M. \& Bunker, C. B. Human papillomavirus in the era of highly active antiretroviral therapy for human immunodeficiency virus: an immune reconstitution-associated disease? Br. J. Dermatol., 162(1):6-11, 2010.

Müller, M.; Wandel, S.; Colebunders, R.; Attia. S.; Furrer, H.; Egger, M. \& IeDEA Southern and Central Africa. Immune reconstitution inflammatory syndrome in patients starting antiretroviral therapy for HIV infection: a systematic review and meta-analysis. Lancet Infect. Dis., 10(4):251-61, 2010.

Ortega, K. L.; Vale, D. A. \& Magalhães, M. H. Impact of PI and NNRTI HAART-based therapy on oral lesions of Brazilian HIV-infected patients. J. Oral Pathol. Med., 38(6):489-94, 2009.

Ray, S.; Fatima, Z. \& Saxena, A. Drugs for AIDS. Mini Rev. Med. Chem., 10(2):147-61, 2010.

Syrjänen, S.; Lodi, G.; von Bültzingslöwen, I.; Aliko, A.; Arduino, P.; Campisi, G.; Challacombe, S.; Ficarra, G.; Flaitz, C.; Zhou, H. M.; Maeda, H.; Miller, C. \& Jontell, M. Human papillomaviruses in oral carcinoma and oral potentially malignant disorders: a systematic review. Oral Dis., 17 Suppl. 1:58-72, 2011.

Taiwo, O. O. \& Hassan, Z. The impact of Highly Active Antiretroviral Therapy (HAART) on the clinical features of HIV - related oral lesions in Nigeria. AIDS Res. Ther., 7:19, 2010.

\author{
Dirección para correspondencia: \\ Dr. Rodrigo Soto Ramírez \\ Fernando Lazcano 1280 \\ Código postal: 8910110 \\ San Miguel \\ Santiago \\ CHILE
}

Email: rodrigo.soto@mayor.cl

Recibido : 07-10-2017

Aceptado: 06-01-2018 